\title{
Relativistic Gravitational Field and the Universe's Figure of a Hyperboloid of Revolution at the Fifth Dimension
}

\section{Adalberto da Costa Dias*}

Darcy Ribeiro Northern Fluminense State University, Rio de Janeiro, Brazil

\begin{abstract}
Says the mass and energy conservation law: mass and energy cannot be created nor destroyed they only can be transformed one into another. The way this transformation is made, according to Einstein's Equation determines the state expansive or contractive of the universe.

Thus, a wave nature process, expansive, explosive and hot occurs when mass is transformed into white energy, like the one that originated the universe in the Big Bang. Such a process, that propagated in decelerated manner (antigravity), created the space and the time, until that the white energy stopped, finalizing the process of expansion in the position of the stars, where occurs a struggle of co-existence between energy and matter.

Then, another wave nature process contractive, implosive and cold occurs when dark energy is transformed into mass, in the periphery of the stars, like the one that bring the universe back to The Big Bang. Such a process that propagates in accelerate manner (gravity), causes the collision between galaxies, until that the mass is accumulate inside the black holes (dark matter).

The space-time-mass deformation caused by relativistic gravitational field corresponds to a hyperboloid of revolution at fifth dimension, lending this shape to the universe. This is the same type of space-time deformation founded by the general relativity theory.

Stars are grouped together forming galaxies by attraction of black holes, which, in turn, the galaxies are grouped together forming the universe by attraction of The Big Hole - the black hole located in the gravitational center of the universe itself.

The Big Hole, at the end of its evolution, reduces to the Hawking point of singularity, storing the whole universal mass in the form of photons, without occuping free spaces (dark matter), and explodes in The Big Bang, cyclically, over and over and over again, indefinitely. The Big Bang explosion is dissociated from nuclear reactions.
\end{abstract}

Keywords: Relativistic gravitational field; Hyperboloid of revolution; Fifth dimension; Dark energy; Dark matter; The big hole

\section{Introduction}

The minimum necessary to be able to write a scientific paper regarding universe's evolution is to be a studious in cosmology. The author, who has such a profile, proposes to contribute to the understanding of the universe's evolution, always respecting the great masters opinion, even if they may seem sometimes misleading $[1,2,3,4]$.

\section{The mass and energy conservation law}

Einstein's Equation [1] must also be considered as one of the mathematical foundations of the mass and energy conservation law. Says this law that, in the universe, mass and energy cannot be created nor destroyed, they only can be transformed one into another. The way this transformation is made, determines the state expansive or contractive of the universe, according to Einstein's Equation (1).

$$
E / c^{2}=m
$$

\section{The universe in contraction}

When dark energy $(E)$ is transformed into mass $(m)$, according to Einstein's Equation (1), where $\left(c^{2}\right)$ is the speed of light squared, a wave nature process contractive, implosive and cold occurs giving rise to the contraction cycle of the universe (Figure 2). Dark energy is linked to the gravity acceleration of black holes. This is the reason why dark energy cannot be mass-transformed in the laboratory, because it is solely a cosmic phenomenon.

\section{Newton's contributions to the gravity acceleration}

According to Newton [4], the gravitational potential $(U)$ (Equation 2) goes to zero at infinity $(s \rightarrow+\infty)$.

$$
U=G \frac{m}{s}
$$

Where: $\left[\mathrm{G}=6.67408 \times 10^{-11}(\text { meter })^{3}(\mathrm{Kg})^{-1}(\mathrm{sec})^{-2}\right]$ is the constant of universal gravitation, $(m)$ is mass, $\left[\mathrm{s}=\left(\mathrm{x}^{2}+\mathrm{y}^{2}+\mathrm{z}^{2}\right)^{\frac{1}{2}}\right]$ is distance straight and $(x, y, z)$ are the three dimensions of space.

The gradient $(\bar{\nabla})$ (Equation 3), which points to infinity (Figure 1), when negative points in the opposite sense, towards the growth of the potential (U) (Equation 2) and to the growth of gravity acceleration $(\bar{g})$ (Equation 4).

*Corresponding author: Adalberto da Costa Dias, Darcy Ribeiro Northern Fluminense State University, Rio de Janeiro, Brazil, Tel : (+55)22-998101038 E-mail: adalbertocostadias@outlook.com

Received September 27, 2017; Accepted October 18, 2017; Published October 25, 2017

Citation: Dias AC (2017) Relativistic Gravitational Field and the Universe's Figure of a Hyperboloid of Revolution at the Fifth Dimension. J Astrophys Aerospace Technol 5: 151. doi:10.4172/2329-6542.1000151

Copyright: (c) 2017 Dias AC. This is an open-access article distributed under the terms of the Creative Commons Attribution License, which permits unrestricted use, distribution, and reproduction in any medium, provided the original author and source are credited. 


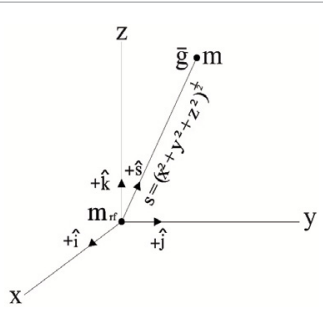

Figure 1: Graphical representation of gravity acceleration $(\bar{g})$ at three dimensions of space (x, y, z) according to Newton's conception (1687). By illustration $(m)$ represents the mass of the apple that fell on the head of Newton and $\left(m_{r}\right)$ represents the mass taken as referece in the gravitational center of the Earth.

$$
\begin{aligned}
& \bar{\nabla}=+\hat{i} \frac{\partial}{\partial x}+\hat{j} \frac{\partial}{\partial y}+\hat{k} \frac{\partial}{\partial z}=+\hat{s} \frac{\partial}{\partial s} \\
& \bar{g}=-\bar{\nabla} U=-G m \hat{s} \frac{\partial}{\partial s}\left(\frac{1}{s}\right)=\frac{G m}{s^{2}} \hat{s}=\frac{\bar{F}_{a}}{m_{r f}} \\
& \text { Where: }\left[\hat{s}=\frac{\bar{s}}{s}=\frac{x \hat{i}+y \hat{j}+z \hat{k}}{\left(x^{2}+y^{2}+z^{2}\right)^{\frac{1}{2}}}\right] .
\end{aligned}
$$

In Newton's words [4] "matter attracts matter, in the direct ratio of the masses ( $m$ times $\left.m_{r f}\right)$ and in the inverse ratio of the distance straight squared $\left(S^{2}\right)$, being $\left(\bar{F}_{a}\right)$ the force vector of the gravitational attraction" (Equation 4).

\section{Inserting Newton's contributions to the gravity acceleration among Einstein's contributions}

Based on the general relativity theory [2], the gravitational potential [4] (Equation 2) grows with decrease of space, also growing with growth of velocity squared $\left(v^{2}\right)$ and, consequently, grows with decrease of time $(\mathrm{t})$, in the fourth dimension $(x, y, z, t)$ (Equation 5).

$$
G \frac{m}{s}=v^{2}(m, s, t)
$$

According to Einstein [2], the relativistic mathematical Equations among the variables $(m, s, t)$ and the variable $\left(v^{2}\right)$ are thus represented (Equations 6, 7, 8 and 9).

$$
\begin{aligned}
& \mathrm{m}=\mathrm{m}_{0}\left(1-\frac{\mathrm{v}_{0}^{2}}{\mathrm{c}^{2}}\right)^{-\frac{1}{2}} \\
& \mathrm{~s}=\mathrm{s}_{0}\left(1-\frac{\mathrm{v}_{0}^{2}}{\mathrm{c}^{2}}\right)^{\frac{1}{2}} \\
& \mathrm{t}=\mathrm{t}_{0}\left(1-\frac{\mathrm{v}_{0}^{2}}{\mathrm{c}^{2}}\right)^{-\frac{1}{2}} \\
& \mathrm{v}^{2}=\mathrm{G} \frac{\mathrm{m}_{0}}{\mathrm{~s}_{0}}\left(1-\frac{\mathrm{v}_{0}^{2}}{\mathrm{c}^{2}}\right)^{-1}
\end{aligned}
$$

The gradient and the relativistic gravitational field at fourth dimension, according to Newton's and Einstein's contributions, are thus represented (Equations 10 and 11):

$$
\begin{aligned}
& \bar{\nabla}=+\hat{i} \frac{\partial}{\partial x}+\hat{j} \frac{\partial}{\partial y}+\hat{k} \frac{\partial}{\partial z}+\hat{t} \frac{\partial}{\partial t}=+\hat{s} \frac{\partial}{\partial s}+\hat{t} \frac{\partial}{\partial t} \\
& \bar{g}=-G m \hat{s} \frac{\partial}{\partial s}\left(\frac{1}{s}\right)-\hat{t} \frac{\partial}{\partial t}\left(v^{2}\right)=v^{2}\left(\frac{\hat{s}}{s}+\frac{\hat{t}}{t}\right)= \\
& =G \frac{m_{0}}{s_{0}}\left(1-\frac{v_{0}^{2}}{c^{2}}\right)^{-\frac{1}{2}}\left[\left(1-\frac{v_{0}^{2}}{c^{2}}\right)^{-1} \frac{\hat{s}}{s_{0}}+\frac{\hat{t}}{t_{0}}\right]=\frac{\bar{F}_{a}}{m_{r f}}
\end{aligned}
$$

\section{Inserting Newton's and Einstein's contributions among Dias' contributions to the gravity acceleration}

According to Dias, Adalberto da Costa (this article), the universe is in the fifth dimension $(x, y, z, t, m)$. When a stone is thrown and breaks a windowpane, the location is characterized in function of $(x, y, z)$, the three dimensions of space, while the fourth dimension $(t)$ represents the occurrence's time. The fifth dimension $\left(m=E / c^{2}\right)$ represents the mass of the material body that broke the windowpane, which been the stone in the specific case.

Thus, the velocity squared (Equation 5 and 9 ) being identified as the independent variable, besides grows with decrease of space (Equations 5 and 7) distance straight $\left[\mathrm{s}=\left(\mathrm{x}^{2}+\mathrm{y}^{2}+\mathrm{z}^{2}\right)^{\frac{1}{2}}\right]$ and growing with decrease of time(Equations 5 and 8), also grows with the growth of the mass accumulation (Equations 5 and 6).

The gradient (Equation 12), when negative is applied to gravitational potential (Equation 5), which mathematically equals relativistic gravitational field (Equation 13), which corresponds to a hyperboloid of revolution at fifth dimension (Figure 2), lending this shape to the universe.

$$
\begin{aligned}
& \bar{\nabla}=+\hat{i} \frac{\partial}{\partial x}+\hat{j} \frac{\partial}{\partial y}+\hat{k} \frac{\partial}{\partial z}+\hat{t} \frac{\partial}{\partial t}-\hat{m} \frac{\partial}{\partial m}= \\
& =+\hat{s} \frac{\partial}{\partial s}+\hat{t} \frac{\partial}{\partial t}-\hat{m} \frac{\partial}{\partial m} \\
& \bar{g}=-G m \hat{s} \frac{\partial}{\partial s}\left(\frac{1}{s}\right)-\hat{t} \frac{\partial}{\partial t}\left(v^{2}\right)+\hat{m} \frac{G}{s}= \\
& =v^{2}\left(\frac{\hat{s}}{s}+\frac{\hat{t}}{t}+\frac{\hat{m}}{m}\right)=\frac{\bar{F}_{a}}{m_{r f}}= \\
& =G \frac{m_{0}}{s_{0}}\left(1-\frac{v_{0}^{2}}{c^{2}}\right)^{-\frac{1}{2}}\left[\left(1-\frac{v_{0}^{2}}{c^{2}}\right)^{-1} \frac{\hat{s}}{s_{0}}+\frac{\hat{t}}{t_{0}}+\frac{\hat{m}}{m_{0}}\right]
\end{aligned}
$$

In the Figure 2, the time straight axis $(t)$ and the mass straight axis $(m)$ make up the fifth dimension with the distance straight axis $\left[s=\left(x^{2}+y^{2}+z^{2}\right)^{\frac{1}{2}}\right]$, which was borrowed from Figure 1.

The relativistic gravitational field $(\bar{g})$ (Equation 13$)$ has its origin in the attraction among masses that gravitate. deforming the space-timemass into a hyperboloid of revolution at fifth dimension (Figure 2).

This is an acceleration phenomenon not related of hot compress that pushes, but rather is coupled to a force vector of gravitational attraction $\overline{F_{a}}$ (Figure 2 and Equation 13) of contractive, implosive and cold nature, that pulls by gravitation the attracted masses $\left(m_{a}\right)$, together 


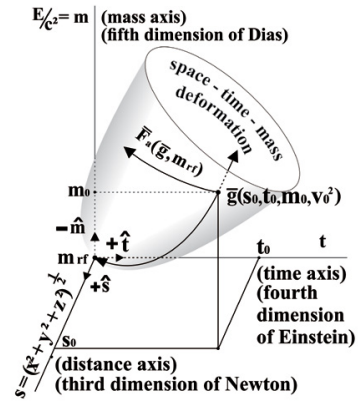

Figure 2: Graphical representation of relativistic gravitational field $(\bar{g})$, which corresponds to a hyperboloid of revolution at fifth dimension according to the conception of Dias, Adalberto da Costa.

with the space and, consequently, with the distance straight $\left(s_{0}\right)$ and with the time $\left(t_{0}\right)$, at the velocity squared $\left(v_{0}^{2}\right)$, towards the center of mass attraction $\left(m_{r f}\right)$, taken as reference.

\section{The solar system}

When $\left(m_{r}\right)$ (Figure 2 and Equation 13$)$ is the mass in the gravitational center of the sun taken as reference and the attracted masses that gravitate $\left(m_{0}\right)$ are planets, it is the solar system.

\section{Black holes}

When $\left(m_{r f}\right)$ (Figure 2 and Equation 13) is the mass in the gravitational center of a black hole taken as reference and the attracted masses that gravitate $\left(m_{0}\right)$ are stars, it is a galaxy.

The Equation (14) mathematically defines the implosive power of a black hole, where (d) is density, $\left(m_{r}\right)$ is the mass in the gravitational center of the black hole taken as reference (dark matter) and $\left(v_{o l}\right)$ is volume:

$$
\lim _{d \rightarrow+\infty} d=\lim _{v_{o l} \rightarrow 0} \frac{m_{r f}}{v_{o l}}
$$

It is not entirely correct to say that, black holes attract the light inclusive, because, the only kind of matter that fits inside black holes are particles of light (photons), which by the reduced volume, tending to zero (Equation 14), are the treshold between mass and energy. The particle of light represents the maximum limit of contraction and velocity of the Universe.

The proof that the only kind of matter that fits inside black holes are particles of light consists of the fact that black holes are always circled by a cold bright cloud inside the galaxies.

Black holes (Figure 2) are able to store in a single point, whithin them, all the particles of light emanating from all the stars contained in the same galaxy.

Even with the colossal implosive power of black holes, causing the volume tending to zero (Equation 14$)$, the mass $\left(m_{r f}\right)$ remains latente within them, because the mass is safeguarded by the mass and energy conservation law.

Based on works developed by Newton [4] and by Einstein [1,2], and according to Figure 2 and Equation (13), when the universe is in contraction:

1. The time count is regressive;

2. The space is implosive;

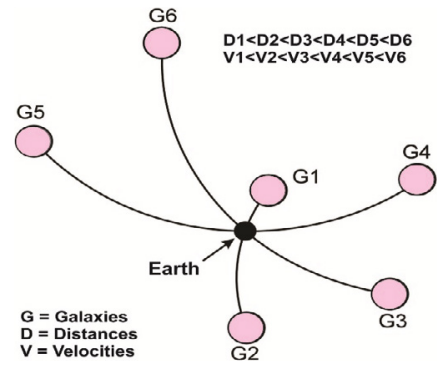

Figure 3: About new interpretations of Hubble's observations [5]

3. The mass is cumulative within black holes (dark matter);

4. The black holes, themselves, are attracted to each other, causing the collision between galaxies, through The Big Crunch process [3].

According to astrophysical research, the universe consists of $70 \%$ of dark energy, $26 \%$ of dark matter and $4 \%$ of observable matter.

Three consequences for the universe is in contraction:

1. The only possibility of dark energy to be mass transformed is by the action of gravity acceleration of black holes. Thus, how much time is needed so that the process of contractive, implosive and cold nature, related to $70 \%$ dark energy, bring the universe back to The Big Bang with the help of gravity acceleration of black holes?

2. Dark matter is formed when photons of light coming from the stars, losing its luminosity, is stored inside the black holes without occupying free spaces, that is, in a volume tending to zero (Equation 14). How long it took for the $26 \%$ of dark matter to be stored inside the black holes?

3. How much time is it still needed for the $4 \%$ of observable matter can be stored inside the black holes?

\section{The Big Hole}

When $\left(m_{r r}\right)$ (Figure 2 and Equation 13) is the mass taken as reference of the black hole called here The Big Hole, located in the gravitational center of the universe and the attracted masses that gravitate $\left(m_{o}\right)$ are galaxies, then it is the universe itself.

It is The Big Hole (Figure 2), which maintains by extreme attraction, the universe unified forming a constellation of galaxies.

If The Big Hole did not exist then the universe would not exist either, because in this case the galaxies would come out of their universal constellation, out of the universe itself, which would be an absurd.

As a consequence of the contracting universe, stars are grouped together forming galaxies (Figure 2) by attraction of black holes, which, in turn, the galaxies are grouped together forming the universe (Figure 2) by attraction of The Big Hole.

The Big Hole has, through The Big Crunch process [3], the capacity to swallow all the black holes of all the galaxies contained in the universe, without occupying free spaces.

The reddish color of the doppler effect observed by Hubble [5] simply means that the galaxies were in front of the point of observation that it was the Earth and moving away from it (Figure 3).

The velocities observed by Hubble [5], very large and everincreasing, as that the galaxies moved away from the Earth (Figure 
3) are the mathematical proof (Equation 13 and Figure 2), that the galaxies were gravitating in a contractive, implosive, and cold fashion, accelerating towards The Big Hole - the black hole located in the gravitational centre of the universe, as a consequence of the universe in contraction.

\section{Back to The Big Bang with the expansion of the universe}

The universe originated from The Big Bang by the transformation of mass into white energy - a wave nature process, expansive, explosive and hot, according to Einstein's Equation (15). The mass in question (dark matter) is one whose volume tend to zero (Equation 14), that is contained within the black hole called here The Big Hole, which reduces to the Hawking [3] point of singularity at the end of universal evolution.

Hawking [3] was correct in saying that the expanding universe originated from a point of singularity. But, what he did not say was that the universal mass really stay contained at that point of singularity (Equation14), because the mass is safeguarded by the mass and energy conservation law.

The wave nature process, expansive, explosive and hot allowed that the wave front of white energy was started from the point of singularity in the speed of light squared. This wave nature process propagated in decelerated manner (antigravity), creating the space and the time, until that the white energy stopped, finalizing the expansion cycle of the universe in the position of the stars, where occurs a struggle of coexistence between energy and matter. The question to be answered here is: how long did it take the universe to expand from The Big Bang until the farthest star?

The Big Bang explosion, which is dissociated from nuclear reactions, occurs due to the non-existence of a second material body, when the universe is reduced to the Hawking [3] point of singularity, according to Figure 2 and Equations (13, 14 and 15).

$$
\lim _{\substack{s_{0} \rightarrow 0 \\ t_{0} \rightarrow 0 \\ m_{0} \rightarrow m_{f f}}} m_{0}=\lim _{v_{v_{0}^{2}}^{2} \rightarrow c^{2}} \frac{E}{v_{0}^{\prime}}
$$

In this specific case, the trigger is fired when the force of gravitational attraction, reversing its vector sense turns into a force of repulsion, for not having a second body to attract, beginning an antigravity cycle for the universe (Equation 16):

$$
-\bar{g}=\bar{\nabla}\left(\mathrm{G} \frac{\mathrm{m}}{\mathrm{s}}\right)=\bar{\nabla}\left(\mathrm{v}^{2}\right)
$$

Finally, a mathematical link between relativistic gravitational field (macrocosm) and quantum mechanics (microcosm) is established through the Equations (13,14 and 15) in the universal figure of a hyperboloid of revolution at fifth dimension (Figure 2).

\section{Conclusions}

Based on works developed by Isaac Newton, Albert Einstein and Stephen Hawking, Dias, Adalberto da Costa says that:

1. In obedience to the mass and energy conservation law, the way mass and energy are transformed into one another determines the state expansive or contractive of the universe.

2. When mass is transformed into white energy, a wave nature process, expansive, explosive and hot occurs, like the one that originated the universe in The Big Bang.
3. When dark energy is transformed into mass, a wave nature process contractive, implosive and cold occurs, like the one that bring the universe back to The Big Bang. Dark energy is linked to the gravity acceleration of black holes. This is the reason why dark energy cannot be mass-transformed in the laboratory, because it is solely a cosmic phenomenon.

4. The time count is regressive, the space is implosive, the mass is cumulative within black holes (dark matter) and the black holes themselves are attracted to each other causing the collision between galaxies because the universe is in contraction.

5. Gravity acceleration is not related of hot compress that pushes, but rather is coupled to a force vector of masses attraction of contractive, implosive and cold nature, that pulls by gravitation the attracted masses, together with the space and, consequently, with the distance straight and with the time, at the velocity squared, towards the center of mass attraction taken as reference.

6. A mathematical link is established between relativistic gravitational field and quantum mechanics in the universal figure of a hyperboloid of revolution at fifth dimension.

7. The space-time-mass deformation caused by relativistic gravitational field corresponds to a hyperboloid of revolution at fifth dimension, lending this shape to the stellar systems, including the sun and its planets, to the galaxies, to the black holes, to The Big Hole, to the universe.

8. It is The Big Hole - the black hole located in the gravitational center of the universe that maintains, by extreme attraction, the universe itself unified forming a constellation of galaxies.

9. The proof that the only kind of matter that fits inside black holes are particles of light consists of the fact that black holes are always circled by a cold bright cloud inside the galaxies.

10. While black holes are able to store, in a single point within them, all the particles of light emanating from all the stars contained in the same galaxy, The Big Hole has the capacity to swallow all the black holes of all the galaxies contained in the universe through The Big Crunch process.

11. The Big Hole reduces to a point of singularity at the end of universal evolution, storing the whole universal mass without occupying free spaces (dark matter) and explodes in The Big Bang, cyclically, over and over and over again, indefinitely.

12. The Big Bang explosion, which is dissociated from nuclear reactions, occurs due to the non-existence of a second material body, when the universe is reduced to a point of singularity. In this specific case, the trigger is fired when the force of gravitational attraction, reversing its vector sense turns into a force of repulsion, for not having a second body to attract, beginning an antigravity cycle for the universe.

13. Even if the distances are the same, but traveled at different velocities; taking into account that the transgressive count of time spent in the expansion process, due to antigravity deceleration, is greater than the regressive count of time spent in the contraction process, due to gravity acceleration, a question arises: what would be the total age of the universe?

14. One consequence of the counting of time being regressive in the contracting universe is that it evolves from the future into the past with increasing velocities. There is a realization that time passes faster each year. My grandfather was born and died in the future and therefore 
Citation: Dias AC (2017) Relativistic Gravitational Field and the Universe's Figure of a Hyperboloid of Revolution at the Fifth Dimension. J Astrophys Aerospace Technol 5: 151. doi:10.4172/2329-6542.1000151

Page 5 of 5

there is no possibility of meeting him and eventually killing him in the past where I am going with the rest of the mankind. The extinsion of dinosaurs occurred in the future because the universe is in contraction.

\section{References}

1. Einstein A (1905) Ist die Trägheit eines Körpers von seinem Energieinhalt abhängig? Annalen der Physik 323: 639-641.

2. Einstein A (1916) Die Grundlage der Allgemeinem Relativitätstheorie. Annalen der Physik 354: 769-822.

3. Hawking S (1971) Gravitational radiation from colliding black holes. Phy Rev Lett 26: 1344-1346.

4. Newton I (1687) Philosophiae naturalis principia mathematica (Mathematical principles of natural philosophy).

5. Hubble E (1929) A relation between distance and radial velocity among extragalactic nebulae. Proceedings of the National Academy of Sciences, USA. 15: 168-173. 